\title{
An Age-Structured Model for the Spread of Epidemic Cholera: Analysis and Simulation
}

\author{
Alen Alexanderian* \\ Matthias K. Gobbert ${ }^{\dagger}$ \\ K. Renee Fister ${ }^{\ddagger}$ \\ Holly Gaff ${ }^{\varsigma}$ \\ Suzanne Lenhart \\ Elsa Schaefer\| \\ May 31, 2011
}

\begin{abstract}
Occasional outbreaks of cholera epidemics across the world demonstrate that the disease continues to pose a public health threat. Traditional models for the spread of infectious diseases are based on systems of ordinary differential equations. Since disease dynamics such as vaccine efficacy and the risk for contracting cholera depend on the age of the humans, an age-structured model offers additional insights and the possibility to study the effects of treatment options. The investigated model is given as a system of hyperbolic (first order) partial differential equations in combination with ordinary differential equations. First, using a representation from the method of characteristics and a fixed point argument, we prove the existence and uniqueness of a solution to our nonlinear system. Then we present a finite difference approximation to the model and study the effect of high and low rates of shedding of cholera vibrios on the dynamics of the spread of the disease. The simulations demonstrate the explosive nature of cholera outbreaks that is observed in reality. The contrast of results for high and low rates of shedding of vibrios suggest a possible underlying cause for this effect.
\end{abstract}

Key words. Epidemic cholera, SIR model, Hyperbolic transport, Finite differences.

AMS subject classifications (2000). 35L45, 35L50, 92D30, 93A30, 74S20, 81T80.

\footnotetext{
*Department of Mechanical Engineering, Johns Hopkins University, Baltimore, MD 21218, USA, aalexa20@jhu.edu

$\dagger$ Corresponding author, Department of Mathematics and Statistics, University of Maryland, Baltimore County, Baltimore, MD 21250, gobbert@umbc.edu, Phone (410) 455-2404, Fax (410) 455-1066

${ }^{\ddagger}$ Department of Mathematics and Statistics, Murray State University, Murray, KY 42071, renee.fister@murraystate.edu

$\S$ Virginia Modeling, Analysis and Simulation Center and the Department of Biological Sciences, Old Dominion University, Norfolk, VA 23929, hgaff@odu.edu

"Department of Mathematics, University of Tennessee, Knoxville, TN 37996, lenhart@math.utk. edu

"Department of Mathematics, Marymount University, Arlington, VA 22207, elsa.schaefer@marymount.edu 


\section{Introduction}

The study of cholera continues to be an important problem in mathematical epidemiology, as increasingly frequent outbreaks of cholera epidemics continue to pose a public health threat $[10,46]$ for humans in crowded conditions with poor sanitation such as in a refugee camp. A common type of model for the spread of an infectious disease is an SIR model, so named after the categorization of individuals in the classes of susceptible, infected, and recovered populations [8]. SIR models of cholera developed so far are time-dependent models which give rise to a system of ordinary differential equations (ODEs); see [10, 11, 22, 24, 30].

In 2001, Codeço published an ODE cholera model which analyzed the interplay between infected humans and the concentration of cholera bacteria in the surrounding environment and the resulting disease dynamics [10]. The next year Merrell and Butler published a finding that freshly shed cholera bacteria from human intestines are 700 times more infectious than bacteria shed only hours previously [27]. Thus, to model the pathway of infection more precisely, Hartley et al. [22] proposed a model which takes into account the role of hyperinfective vibrios introduced into the water reserves by the infected people in the population; this new model explains the explosive nature of the disease more clearly as seen in historical accounts of cholera epidemics. This feature of hyperinfective vibrios, which gives increased transmission, will be included in our model. See [18, 42] for biological sequencing background for this pathogen.

Note that King et al. [24] proposed a two-patch cholera ODE model including classes for 'mild' infections and the feature of waning immunity. The work by Miller Neilan et al. [30] investigated optimal control of three strategies to slow the spread of the disease in ODE model with hyperinfectious vibrios, asymptomatic infecteds and waning immunity. There is another modeling approach with a compartment that tracks pathogen in the water; this approach gives a SIWR system of four ODEs and was used to simulate cholera in the 19th century in London, [40, 41].

We now work on a model combining features of models in Codeço and Hartley et al. $[10,22]$ to the next stage, but providing a new feature by taking into account the influence of the age of the affected humans. People of different ages have different pathologies of infection and of treatment. Although using age-structured models to study the spread of infectious diseases is not in itself a new idea, the application of such a model in the study of cholera will provide novel insights. At the heart of this age-structured model is a coupled system of hyperbolic partial differential equations (PDEs) which model the human dynamics in a cholera outbreak. The equations which track the growth of the cholera bacteria will not need age structure and thus remain as ODEs. While the introduction of a system of PDEs in place of a system of ODEs enhances the interconnectivity and accuracy of the model, the resulting system of equations is substantially more complicated to analyze. The numerical computation needed to produce outbreak simulations becomes a nontrivial task which introduces new challenges not encountered in the traditional ODE models. It is additionally nontrivial to establish existence and uniqueness of solutions to the model system.

For further background of age-structured models, we encourage the reader to see $[2,3$, $5,12,13,45]$. For equilibrium analysis of an age-structured competition model, see [20, 36]. The existence results and formulation of age-structured models were introduced by Gurtin 
and MacCamy [21] and Venturino [43]. Barbu and Iannelli [6] studied the mathematical theory behind age-structured populations. Brauer [9], Li [26], and Saleem [36] provided insight into nonlinear age-dependent populations and predator prey dynamics. Fister and Lenhart [17] investigated the existence of solutions to a predator prey age-structured model with control. For further background of epidemic models including age structure, spatial features, and impulse effects, see [25, 44, 47, 4, 38, 14].

This paper will develop an age-structured cholera model and provide a theoretical and numerical analysis of the model. In Section 2, we will discuss the system of differential equations along with initial and boundary conditions that form our disease model. In Section 3, we prove the existence and uniqueness of a solution in $L^{1}$ and $L^{\infty}$ to our PDE system using a fixed point argument on a representation derived from the method of characteristics. We discuss the numerical method implemented in Section 4; and subsequently in Section 5, we discuss the numerical experiments conducted and present our computational results along with their practical interpretations. We provide concluding remarks in Section 6.

\section{The Model}

In this section, we describe the system of differential equations which describes our cholera model. We consider a given human population which we divide into three categories: susceptible, infected, and recovered. For each category, we consider both the age density and the population changes over time. Thus, we write the number of humans in each category $S=S(a, t), I=I(a, t)$, and $R=R(a, t)$ as functions of age $a$ and time $t$. In a theoretical discussion, perhaps units of variables in equations are not crucial because one knows that everything can be balanced with a suitable coefficient. However, for real-life computations on concrete problems, one needs to pay close attention to the units of the model quantities. To make a dimensional study of the model simpler, we formally use units of weeks for the age of humans $a$ and days for the simulation time $t$ in the tables of this paper, though we also use conventional units of years in the text when that makes the intention of the formulas clearer. With concentration units of humans per age for the population density variables, following, e.g., [23], the number of susceptible people between, for instance, ages $a_{1}$ and $a_{2}$ at a time $t$ is given by $\int_{a_{1}}^{a_{2}} S(a, t) d a$. Specifically, to find the number of susceptible humans of

a particular age $a_{0}$ years, we compute $\int_{a_{0}}^{a_{0}+1} S(a, t) d a$ using the conventional understanding that all humans from $a=a_{0}$ years to $a=a_{0}+1$ years are considered $a_{0}$ years old. Analogous approaches are used for infected and recovered humans $I(a, t)$ and $R(a, t)$. Following Hartley et al. [22], we have two vibrios compartments, and we denote the hyperinfective and non-hyperinfective cholera bacteria by $B_{H}=B_{H}(t)$ and $B_{L}=B_{L}(t)$, respectively. The five quantities $S, I, R, B_{L}$, and $B_{L}$ are the dependent variables of the model.

The model is pictured in Figure 1. The majority of microparasite diseases are directly transmitted by close contact with an infectious individual, but cholera is usually considered as waterborne. To account for the various factors affecting the dynamics of a cholera epidemic, we have introduced additional coefficient functions, which may be constant, or may vary with age or time (or both). We assume a setting of a refugee camp, and thus human demographics include a recruitment term $\Lambda(a, t)$ along with a natural death rate $b(a)$. The susceptible 


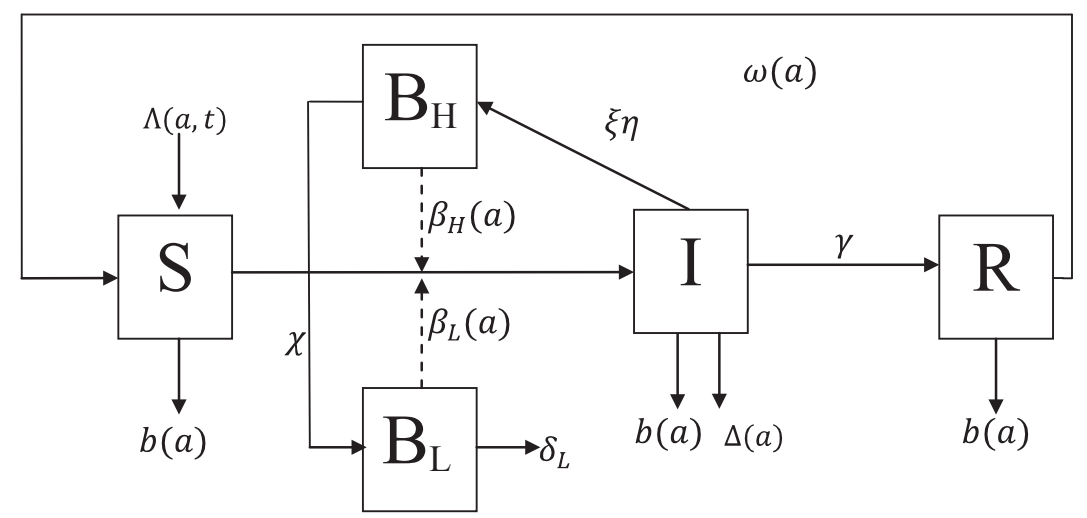

Figure 1: Diagram of cholera dynamics.

individuals become infected as a result of ingesting bacteria at rates $\beta_{H}(a)$ and $\beta_{L}(a)$, with the bacteria concentrations measured with respect to their infectious doses, $\kappa_{H}(a)$ and $\kappa_{L}(a)$. Individuals recover from cholera at rates $\gamma_{1}$ for untreated cholera and $\gamma_{2}$ for treated cholera. These are denoted as just $\gamma$ in Figure 1. Based on studies that confirm age-dependent length of immunity from vaccine trials, we assume similarly that recovered individuals gain short-term immunity that wanes at a rate $w(a)$ which depends on age $[15,32,33]$. Infected humans contribute to the concentration of cholera bacteria in the local water supply at a rate $\xi \eta$ which accounts for human shedding rates as well as the relative local impact, and is explained more fully in the discussion following the model equations. Finally, we assume that infected humans die as a result of cholera at an age-based rate $\Delta(a)$. The bacteria move from the hyperinfectious state, $B_{H}$, to a less-infectious class $B_{L}$ at a rate of $\chi$. The less-infectious bacteria experience a natural removal rate of $\delta_{L}$ due to death or predation. See Table 1 for a complete description of the model quantities and their units.

There are various strategies that can be implemented in a cholera outbreak to either decrease the numbers becoming infected or to decrease the loss of life. In this paper, we include two strategies $u(a, t)$ and $h(a, t)$ that represent antibiotic treatment and hydration therapy, respectively. The antibiotic treatment lessens the duration and quantity of an infected human's contribution to the concentration of bacteria in the environment, while hydration therapy saves lives without lessening an infected individual's contribution to the environment. We note that in reality antibiotic treatment would not be administered without hydration therapy, and that additionally there are great concerns of the spread of resistant bacteria as a result of antibiotic therapy [35, 28, 29]. However, this investigation provides a first step in developing the numerical and theoretical tools to consider a model with more complex control methods that may more accurately reflect present practices. Additionally, we are investigating the existence of a solution to this nonlinear system in connection with numerical concepts.

We consider the age-time domain, $Q=(0, A) \times(0, T)$, with interventions $(u, h)$ in

$$
\Gamma=\left\{(u, h) \in\left(L^{\infty}(Q)\right)^{2} \mid 0 \leq u(a, t) \leq N_{1}, \quad 0 \leq h(a, t) \leq N_{2} \quad \text { a.e. in } Q\right\},
$$


where $N_{1}<1$ and $N_{2}<1$ denote maximum fractions of intervention. The variables, $\left(S, I, R, B_{H}, B_{L}\right)$, then satisfy the state system:

$$
\begin{aligned}
\frac{\partial S}{\partial t}+\alpha \frac{\partial S}{\partial a}= & \Lambda(a, t)-\beta_{L}(a) \frac{B_{L}(t)}{\kappa_{L}(a)+B_{L}(t)} S(a, t)-\beta_{H}(a) \frac{B_{H}(t)}{\kappa_{H}(a)+B_{H}(t)} S(a, t) \\
& -b(a) S(a, t)+\omega(a) R(a, t) \\
\frac{\partial I}{\partial t}+\alpha \frac{\partial I}{\partial a}= & \beta_{L}(a) \frac{B_{L}(t)}{\kappa_{L}(a)+B_{L}(t)} S(a, t)+\beta_{H}(a) \frac{B_{H}(t)}{\kappa_{H}(a)+B_{H}(t)} S(a, t)-b(a) I(a, t) \\
& -(1-h(a, t)) \Delta(a) I(a, t)-\gamma_{1}(1-u(a, t)) I(a, t)-\gamma_{2} u(a, t) I(a, t) \\
\frac{\partial R}{\partial t}+\alpha \frac{\partial R}{\partial a}= & \gamma_{1}(1-u(a, t)) I(a, t)+\gamma_{2} u(a, t) I(a, t)-b(a) R(a, t)-\omega(a) R(a, t) \\
\frac{d B_{H}}{d t}= & \int_{0}^{A} \xi \eta I(a, t) d a-\chi B_{H}(t) \\
\frac{d B_{L}}{d t}= & \chi B_{H}(t)-\delta_{L} B_{L}(t) .
\end{aligned}
$$

Note that the vibrios interact with the susceptibles with a Monod or Michaelis-Menton type coefficient and the the transmission coefficient is different for $B_{H}$ and $B_{L}$. In the above

equations, $\alpha=\frac{1}{7} \frac{\text { week }}{\text { days }}$ is a coefficient introduced to balance the units of age $a$ in weeks and time $t$ in days. Also, $A$ which appears in the equation for $B_{H}$ is an upper bound on the age of people in the model (we used $A=72$ years in our simulations). The model above incorporates the two control strategies into the model description given previously and in Figure 1. Hydration therapy $h(a, t)$ decreases the percent $\Delta(a)$ of the infected individuals who die from cholera. In the infected class (class I), the multiplicative factors $\gamma_{1}(1-u)$ and $\gamma_{2} u$ represent the rates of recovery for the individuals who have had no antibiotic treatment and for those who have had such a treatment, respectively. The integral term in the $B_{H}$ equation depicts the total number of vibrios that are shed at a rate of $\xi$ with the relative amount of stool $\eta$ per time for an infected individual of age, $a$. This model system is mixed in nature because it involves both hyperbolic first order partial differential equations and ordinary differential equations.

\section{The Boundary and Initial Conditions}

We consider cholera disease dynamics to determine conditions for $S, I$, and $R$. We know that cholera does not transmit vertically from mother to child, and there is evidence that infants have immunity either due to breast-feeding or from the mother, [7, 16, 31, 37]. Several studies have established low disease rates in infants, due perhaps to breastfeeding or crossprotection from Escherichia coli infections that are caused by a similar toxin, [19, 34]. Thus, newborns will appear in the $R$ class. We note that the immunity against infection at time of birth makes this model different from other age-structured SIR models of infectious diseases. We also assume that women between the ages of 15 and 45 give birth to 3 children. These 
considerations translate to the boundary conditions

$$
\begin{aligned}
S(0, t) & =0 \\
I(0, t) & =0 \\
R(0, t) & =\int_{0}^{A}(S(a, t)+I(a, t)+R(a, t)) f(a) d a,
\end{aligned}
$$

where the fecundity function $f$ is modeled as

$$
f(a)= \begin{cases}\frac{1}{5} \sin ^{2}\left[\left(\frac{a-15}{30}\right) \pi\right], & 15<a<45 \\ 0, & \text { otherwise }\end{cases}
$$

This fecundity function is stated here in units of years for clarity, though the code and Table 1 use units of weeks. The assumption that from age 15 to 45 years a woman will generally give birth to three children expresses itself in the integral

$$
\int_{0}^{A} f(a) d a=3
$$

where $A$ is the largest age allowed in the model (which is always greater than 45 years). The assumption of 3 children per woman appears applicable to the countries in the developing world, where cholera is more likely to occur than in Western industrialized nations, where this number tends to be lower than 3 .

The initial conditions are given by

$$
S(a, 0)=S_{0}(a), \quad I(a, 0)=I_{0}(a), \quad R(a, 0)=R_{0}(a), \quad B_{L}(0)=B_{L 0}, \quad B_{H}(0)=B_{H 0} .
$$

We have listed all model parameters with brief descriptions in Table 1. To make things clear, we have also stated the units for each of the quantities.

\section{Existence of the Solution to the State System}

For the existence of a solution, we first must develop a solution determined by the method of characteristics, [5, Section 4.3] and [45, Section 1.3]. Then we prove the existence and uniqueness of solutions using the Banach contraction mapping principle. To find the solution representation for the system (2.2)-(2.6), we introduce the following notation for the right 


\begin{tabular}{|c|c|c|}
\hline Quantity & Description & Units \\
\hline$a$ & age & weeks \\
\hline$t$ & time & days \\
\hline$S(a, t)$ & $\begin{array}{l}\text { susceptible humans of age } a \text { at time } t \\
\text { divided uniformly over all ages }\end{array}$ & $\frac{\text { human }}{\text { week }}$ \\
\hline$I(a, t)$ & infected and infectious humans of age $a$ at time $t$ & $\frac{\text { human }}{\text { week }}$ \\
\hline$R(a, t)$ & removed and immune humans of age $a$ at time $t$ & $\frac{\text { human }}{\text { week }}$ \\
\hline$B_{H}(t)$ & HI vibrio population & cells $/ \mathrm{ml}$ \\
\hline$B_{L}(t)$ & non-HI vibrio population & cells $/ \mathrm{ml}$ \\
\hline$\alpha$ & proportionality factor (wave speed) & week / days \\
\hline$\Lambda(a, t)$ & $\begin{array}{l}\text { recruitment rate, number of susceptible humans } \\
\text { entering population of age } a \text { at time } t\end{array}$ & $\frac{\text { human }}{\text { week-day }}$ \\
\hline$h(a, t)$ & $\begin{array}{l}\text { oral rehydration therapy, reduces disease } \\
\text { related mortality ( } 90 \% \text { effective) of age } a \text { at time } t\end{array}$ & none \\
\hline$u(a, t)$ & antibiotic treatment rate for humans of age $a$ at time $t$ & none \\
\hline$\beta_{L}(a)$ & ingestion rate of non-HI vibrios at age $a$ & 1/day \\
\hline$\beta_{H}(a)$ & ingestion rate of HI vibrios at age $a$ & 1/day \\
\hline$\kappa_{L}(a)$ & saturation constant of non-HI vibrios at age $a$ & cells $/ \mathrm{ml}$ \\
\hline$\kappa_{H}(a)$ & saturation constant of HI vibrios at age $a$ & cells $/ \mathrm{ml}$ \\
\hline$b(a)$ & natural mortality rate of humans at age $a$ & 1/day \\
\hline$\omega(a)$ & rate of waning immunity of humans at age $a$ & $1 /$ day \\
\hline$\Delta(a)$ & disease related mortality rate for humans at age $a$ & 1/day \\
\hline$f(a)$ & maternity rate & $1 /$ week \\
\hline$\gamma_{1}$ & recovery rate of untreated cholera & 1/day \\
\hline$\gamma_{2}$ & recovery rate of treated cholera & 1/day \\
\hline$\xi$ & $\begin{array}{l}\text { rate of shedding of cholera vibrios } \\
\text { from infected human of age } a\end{array}$ & $\frac{\text { cells }}{\text { ml-day-human }}$ \\
\hline$\eta$ & relative amount of stool per time & (no dimension) \\
\hline$\chi$ & rate of vibrio moving from HI to non-HI state & $1 /$ day \\
\hline$\delta_{L}$ & death rate of vibrio in the environment & $1 /$ day \\
\hline
\end{tabular}

Table 1: Model parameters and their units (HI denotes hyperinfectious). 
hand sides of the partial differential equations (PDEs):

$$
\begin{aligned}
& f_{1}\left(B_{L}(t), B_{H}(t), S(a, t), R(a, t)\right)=\Lambda(a, t)-\beta_{L}(a) \frac{B_{L}(t)}{\kappa_{L}(a)+B_{L}(t)} S(a, t) \\
& \quad-\beta_{H}(a) \frac{B_{H}(t)}{\kappa_{H}(a)+B_{H}(t)} S(a, t)+\omega(a) R(a, t), \\
& f_{2}\left(B_{L}(t), B_{H}(t), S(a, t), I(a, t), h(a, t), u(a, t)\right)=\beta_{L}(a) \frac{B_{L}(t)}{\kappa_{L}(a)+B_{L}(t)} S(a, t) \\
& \quad+\beta_{H}(a) \frac{B_{H}(t)}{\kappa_{H}(a)+B_{H}(t)} S(a, t) \\
& \quad-(1-h(a, t)) \Delta(a) I(a, t)-\gamma_{1}(1-u(a, t)) I(a, t)-\gamma_{2} u(a, t) I(a, t), \\
& f_{3}(S(a, t), I(a, t), R(a, t), u(a, t))=\gamma_{1}(1-u(a, t)) I(a, t) \\
& \quad+\gamma_{2} u(a, t) I(a, t)-\omega(a) R(a, t) .
\end{aligned}
$$

Please note that the $b(a) S(a, t), b(a) I(a, t)$ and the $b(a) R(a, t)$ terms are not included in the $f_{i}$ for $i=1,2,3$ terms. They are included in the left hand side of the three partial differential equations (2.2)-(2.4) for use in the representation of the solution via method of characteristics.

Let $M$ be chosen such that

$$
\begin{aligned}
& 0 \leq S_{0}(a), I_{0}(a), R_{0}(a) \text { a.e. } \\
& \int_{0}^{A} S_{0}(a) d a \leq M, \int_{0}^{A} I_{0}(a) d a \leq M, \int_{0}^{A} R_{0}(a) d a \leq M \\
& \text { and } 0 \leq B_{H 0}, B_{L 0} \leq M .
\end{aligned}
$$

We define our state solution space as

$$
\begin{aligned}
X=\left\{\left(S, I, R, B_{H}, B_{L}\right) \in\right. & \left(L^{\infty}\left(0, T ; L^{1}(0, A)\right)\right)^{3} \times\left(L^{\infty}(0, T)\right)^{2} \mid \\
& \sup _{t} \int_{0}^{A}|S(a, t)| d a \leq 2 M, \sup _{t} \int_{0}^{A}|I(a, t)| d a \leq 2 M, \\
& \left.\sup _{t} \int_{0}^{A}|R(a, t)| d a \leq 2 M,\left|B_{H}(t)\right| \leq 2 M,\left|B_{L}(t)\right| \leq 2 M \text { a.e. } t\right\} .
\end{aligned}
$$

Using the method of characteristics, we can find the representation of the solution (if it exists) and we use that representation to build the map to use in our fixed point argument for existence and uniqueness. Next we define a map

$$
\begin{gathered}
L: X \rightarrow X \text { such that } \\
L\left(S, I, R, B_{H}, B_{L}\right)=\left(\begin{array}{l}
L_{1}\left(S, I, R, B_{H}, B_{L}\right), L_{2}\left(S, I, R, B_{H}, B_{L}\right), L_{3}\left(S, I, R, B_{H}, B_{L}\right), \\
\left.L_{4}\left(S, I, R, B_{H}, B_{L}\right), L_{5}\left(S, I, R, B_{H}, B_{L}\right)\right)
\end{array}\right.
\end{gathered}
$$


where $L_{1}$ is associated with equation (2.2) and $L_{2}$ is associated with equation (2.3), etc. and where

$$
L_{1}\left(S, I, R, B_{H}, B_{L}\right)(a, t)=\left\{\begin{array}{l}
e^{-\int_{0}^{t} b(\alpha \tau-\alpha t+a) d \tau} S_{0}(a-\alpha t) \\
+\int_{0}^{t} e^{-\int_{s}^{t} b(\alpha \tau-\alpha t+a) d \tau} \times \\
\left(f _ { 1 } \left(B_{H}(s), B_{L}(s), S(\alpha s+a-\alpha t, s)\right.\right. \\
R(\alpha s+a-\alpha t, s))) d s \\
\text { if } a>\alpha t \\
\\
\left(\frac{1}{\alpha}\right) \int_{0}^{a} e^{-\int_{s}^{a} \frac{b(\tau)}{\alpha} d \tau} \times \\
f_{1}\left(B_{H}\left(\frac{s+\alpha t-a}{\alpha}\right), B_{L}\left(\frac{s+\alpha t-a}{\alpha}\right)\right. \\
\left.S\left(s, \frac{s+\alpha t-a}{\alpha}\right), R\left(s, \frac{s+\alpha t-a}{\alpha}\right)\right) d s \\
\text { if } a<\alpha t
\end{array}\right.
$$

$L_{2}\left(S, I, R, B_{H}, B_{L}\right)(a, t)=\left\{\begin{array}{l}e^{-\int_{0}^{t} b(\alpha \tau-\alpha t+a) d \tau} I_{0}(a-\alpha t) \\ +\int_{0}^{t} e^{-\int_{s}^{t} b(\alpha \tau-\alpha t+a) d \tau} \times \\ f_{2}\left(B_{H}(s), B_{L}(s), S(\alpha s+a-\alpha t, s),\right. \\ I(\alpha s+a-\alpha t, s), h(\alpha s+a-\alpha t, s), u(\alpha s+a-\alpha t, s) d s \\ \text { if } a>\alpha t \\ \left(\frac{1}{\alpha}\right) \int_{0}^{a} e^{-\int_{s}^{a} \frac{b(\tau)}{\alpha} d \tau} \times \\ f_{2}\left(B_{H}\left(\frac{s+\alpha t-a}{\alpha}\right), B_{L}\left(\frac{s+\alpha t-a}{\alpha}\right),\right. \\ \left.S\left(s, \frac{s+\alpha t-a}{\alpha}\right), I\left(s, \frac{s+\alpha t-a}{\alpha}\right), h\left(s, \frac{s+\alpha t-a}{\alpha}\right), u\left(s, \frac{s+\alpha t-a}{\alpha}\right)\right) d s \\ \text { if } a<\alpha t,\end{array}\right.$

$$
L_{3}\left(S, I, R, B_{H}, B_{L}\right)(a, t)=\left\{\begin{array}{l}
e^{-\int_{0}^{t} b(\alpha \tau-\alpha t+a) d \tau} R_{0}(a-\alpha t) \\
+\int_{0}^{t} e^{-\int_{s}^{t} b(\alpha \tau-\alpha t+a) d \tau} \times \\
f_{3}(S(\alpha s+a-\alpha t, s), I(\alpha s+a-\alpha t, s), \\
R(\alpha s+a-\alpha t, s), u(\alpha s+a-\alpha t, s)) d s \\
\text { if } a>\alpha t \\
e^{-\int_{0}^{a} \frac{b(\tau)}{\alpha} d \tau \times} \\
\int_{0}^{A}\left[S\left(s, \frac{\alpha t-a}{\alpha}\right)+I\left(s, \frac{\alpha t-a}{\alpha}\right)+R\left(s, \frac{\alpha t-a}{\alpha}\right)\right] f(s) d s \\
\left.+\left(\frac{1}{\alpha}\right) \int_{0}^{a} e^{-\int_{s}^{a} \frac{b(\tau)}{\alpha} d \tau \times} \times d, \frac{s+\alpha t-a}{\alpha}\right), \\
\left.f_{3}\left(S\left(s, \frac{s+\alpha t-a}{\alpha}\right), I\left(s, \frac{s+\alpha t-\alpha}{\alpha}\right)\right)\right) d s \\
R\left(s, \frac{s+\alpha t-a}{\alpha}\right), u\left(s, \frac{s+\alpha t-a}{\alpha}\right) \\
\text { if } a<\alpha t,
\end{array}\right.
$$

with

$$
L_{4}\left(S, I, R, B_{H}, B_{L}\right)(t)=B_{H 0} e^{-\chi t}+\int_{0}^{t} \int_{0}^{A} e^{-\chi(t-s)} \xi \eta I(a, t) d a d s
$$


and

$$
L_{5}\left(S, I, R, B_{H}, B_{L}\right)(t)=B_{L 0} e^{-\delta_{L} t}+\int_{0}^{t} \chi e^{-\delta_{L}(t-s)} B_{H}(s) d s .
$$

A fixed point of the map $L$, satisfying

$$
\left(S, I, R, B_{H}, B_{L}\right)=\left(L_{1}, L_{2}, L_{3}, L_{4}, L_{5}\right)\left(S, I, R, B_{H}, B_{L}\right)
$$

with each of $S(a, t), I(a, t), R(a, t), B_{H}(t)$, and $B_{L}(t)$ being non-negative, will be a solution $\left(S, I, R, B_{H}, B_{L}\right)=\left(S, I, R, B_{H}, B_{L}\right)(u, h)$ to the state system.

Theorem 3.1 (Existence and uniqueness of solutions) For $(u, h) \in \Gamma$ as defined in (2.1) and $T$ sufficiently small, there exists a unique solution $\left(S, I, R, B_{H}, B_{L}\right)$ to the state system (2.2)-(2.6) with boundary and initial conditions (2.7)-(2.9) and (2.12).

Proof: We prove that the map

$$
L: X \rightarrow X
$$

defined above, is a strict contraction. We note that the functions $f_{1}, f_{2}$ and $f_{3}$ in the $S, I, R$ equations are Lipschitz in their arguments with the Lipschitz constants depending on coefficients and parameters from the model as well as on $M$, through the bounds on $S, I, R$ from the set $X$.

To show that $L$ maps $X$ into $X$, from the definition of the map $L$, the definition of the $L_{i}$ functions for $i=1,2,3$ gives

$$
\int_{0}^{A}\left|L_{i}\left(S, I, R, B_{H}, B_{L}\right)\right|(a, t) d a \leq C_{1} M T+M \leq 2 M
$$

where the single $M$ in the first inequality comes from the bound of $\int_{0}^{A} S_{0}(a) d a, \int_{0}^{A} I_{0}(a) d a$, or $\int_{0}^{A} R_{0}(a) d a$, respectively for $i=1,2,3$. Since $\mathrm{T}$ is sufficiently small, then the above estimate is less than or equal to $2 M$.

Additionally, for $j=4,5$, we have

$$
\left|L_{j}\left(S, I, R, B_{H}, B_{L}\right)\right| \leq \max \left\{B_{H 0}, B_{L 0}\right\}+C_{2} M T \leq 2 M .
$$

The constants $C_{1}$ and $C_{2}$ depend on the coefficients and the parameters in the model. Again, for T sufficiently small, we obtain the estimate above and therefore, we have that the $L$ maps $X$ into $X$.

For the contraction property, for $i=1,2,3$, we consider

$$
\int_{0}^{A}\left|L_{i}\left(S_{1}, I_{1}, R_{1}, B_{H 1}, B_{L 1}\right)-L_{i}\left(S_{2}, I_{2}, R_{2}, B_{H 2}, B_{L 2}\right)\right|(a, t) d a .
$$

In order to proceed, we need to investigate terms such as $\beta_{L}(a) \frac{B_{L}(t)}{\kappa_{L}+B_{L}(t)} S(a, t)$ and in particular their differences. For example, we have

$$
\frac{B_{L 1}(t)}{\kappa_{L}+B_{L 1}(t)}\left|S_{1}-S_{2}\right|(a, t)+\frac{S_{2}(a, t) \kappa_{L}\left|B_{L 1}-B_{L 2}\right|(t)}{\left(\kappa_{L}+B_{L 1}(t)\right)\left(\kappa_{L}+B_{L 2}(t)\right)}
$$


to consider from equations (3.5) and (3.6). For specificity, we demonstrate an estimate of such a term for $a>\alpha t$ in $L_{1}\left(S, I, R, B_{H}, B_{L}\right)(a, t)$.

$$
\begin{aligned}
\int_{0}^{A} \int_{0}^{t} \quad & \mid e^{-\int_{s}^{t} b(\alpha \tau-\alpha t+a) d \tau} \times \\
& \beta_{L}(\alpha s+a-\alpha t, s) \frac{B_{L 2}(s)}{\kappa_{L}+B_{L 2}(s)}\left|S_{2}-S_{1}\right|(\alpha s+a-\alpha t, s) \\
& +\frac{S_{1}(\alpha s+a-\alpha t, s) \kappa_{L}\left|B_{L 2}-B_{L 1}\right|(s)}{\left(\kappa_{L}+B_{L 1}(s)\right)\left(\kappa_{L}+B_{L 2}(s)\right)} \\
+ & \beta_{H}(\alpha s+a-\alpha t, s) \frac{B_{H 2}(s)}{\kappa_{H}+B_{H 2}(s)}\left|S_{2}-S_{1}\right|(\alpha s+a-\alpha t, s) \\
+ & \frac{S_{1}(\alpha s+a-\alpha t, s) \kappa_{H}\left|B_{H 2}-B_{H 1}\right|(s)}{\left(\kappa_{H}+B_{H 1}(s)\right)\left(\kappa_{H}+B_{H 2}(s)\right)} \\
+ & \omega(\alpha s+a-\alpha t, s)\left(R_{2}-R_{1}\right)(\alpha s+a-\alpha t, s) \\
& -v(\alpha s+a-\alpha t, s)\left(S_{2}-S_{1}\right)(\alpha s+a-\alpha t, s) \mid d s d a
\end{aligned}
$$

If we let $s_{1}=\alpha(s-t)+a$ and $s_{2}=s$, then $0<-\alpha t+a<\alpha(s-t)+a<a$ or $0<\alpha(s-t)+a<A$ and $0 \leq s_{2}<T$. In addition, the Jacobian for this transformation is finite. Therefore, we can bound the estimate above by

$$
\begin{aligned}
& \int_{0}^{T} \int_{0}^{A} C_{3}\left|S_{2}-S_{1}\right|\left(s_{1}, s_{2}\right)+C_{4}\left|R_{2}-R_{1}\right|\left(s_{1}, s_{2}\right) d s_{1} d s_{2} \\
+ & C_{5} \int_{0}^{T} \int_{0}^{A}\left|S_{1}\left(s_{1}, s_{2}\right)\right| d s_{1}\left\{\left|B_{L 2}\left(s_{2}\right)-B_{L 1}\left(s_{2}\right)\right|+\left|B_{H 2}\left(s_{2}\right)-B_{H 1}\left(s_{2}\right)\right| d s_{2}\right\} \\
\leq & C_{6} T \sup _{t} \int_{0}^{A}\left(\left|S_{2}-S_{1}\right|+\left|R_{2}-R_{1}\right|\right)(a, t) d a \\
+ & C_{7} M T \sup _{t}\left[\mid\left(B_{L 2}-B_{L 1}|(t)+| B_{H 2}-B_{H 1} \mid(t)\right]\right.
\end{aligned}
$$

where we have replaced the variables $s_{1}$ and $s_{2}$ by $a$ and $t$, respectively. Also, the constants $C_{k}$ for $k=3, \ldots, 7$ depend on the bounds of the coefficients. Moreover, for terms involving the fractional components, we have used the $2 M$ bound for the terms involving the $S_{i}$ for $i=1,2$ in the second term of $(3.10)$ for integrals over $(0, A) \times(0, t)$ when $a>\alpha t$ or for integrals over $(0, A) \times(0, a)$ when $a<\alpha t<\alpha T$. We can gather these estimates to give

$$
\begin{gathered}
\int_{0}^{A}\left|L_{1}\left(S_{1}, I_{1}, R_{1}, B_{H 1}, B_{L 1}\right)-L_{1}\left(S_{2}, I_{2}, R_{2}, B_{H 2}, B_{L 2}\right)\right|(a, t) d a \\
\leq C_{8} T \sup _{t} \int_{0}^{A}\left(\left|S_{2}-S_{1}\right|+\left|R_{2}-R_{1}\right|\right)(a, t) d a+C_{9} M T \sup _{t}\left[\left|B_{L 2}-B_{L 1}\right|(t)+\left|B_{H 2}-B_{H 1}\right|(t)\right] .
\end{gathered}
$$


A similar estimate holds for $j=2,3$.

For $\mathrm{j}=4,5$, we have that

$$
\begin{aligned}
& \left|L_{j}\left(S_{1}, I_{1}, R_{1}, B_{H 1}, B_{L 1}\right)-L_{j}\left(S_{2}, I_{2}, R_{2}, B_{H 2}, B_{L 2}\right)\right|(t) \\
\leq & T \sup _{t} \int_{0}^{A}\left|I_{1}-I_{2}\right|(a, t) d a+C_{10} T \sup _{t}\left|B_{H 1}-B_{H 2}\right|(t),
\end{aligned}
$$

where $C_{10}$ depends on $\chi$ and $\delta$.

By combining the work above and by selecting T sufficiently small, we obtain the contraction result and thus the desired fixed point to the system (2.2)-(2.6).

Remark: By using an argument as Chapter 2 in Webb [45] and noticing that the right hand side of the differential equations has a common factor $S, I, R$, respectively, one can obtain the non-negativity of the solutions.

\section{Numerical Concepts}

In this section, we describe briefly the numerical method used in our computations. The equations for the quantities $S, I$, and $R$ from (2.2)-(2.4) form a hyperbolic system of PDEs; coupled with these, we have the two ODEs for $B_{H}$ and $B_{L}$ from (2.5)-(2.6). Our choice of numerical method is a forward time/backward space finite difference scheme [39, Chapter 1]. For the convenience of the reader we recall the scheme for the simpler case of the scalar one-way wave equation

$$
\frac{\partial w}{\partial t}+\alpha \frac{\partial w}{\partial x}=g(x, t)
$$

where $\alpha$ is a constant (physically the wave speed), and $t$ and $x$ represent time and space, respectively. The forward time/backward space scheme [39] for the above problem is given by

$$
\frac{u_{m}^{n+1}-u_{m}^{n}}{\Delta t}-\alpha \frac{u_{m}^{n}-u_{m-1}^{n}}{\Delta x}=g\left(x_{m}, t_{n}\right),
$$

where $n$ denotes the time index and $m$ the space index in the time and space grid.

We also recall that any explicit scheme is only conditionally stable [39]. To ensure stability of the scheme, a necessary and sufficient condition is the famous Courant-Friedrichs-Lewy (CFL) condition [39, Theorem 1.6.1] which requires

$$
\left|\alpha \frac{\Delta t}{\Delta x}\right| \leq 1
$$

For a given spatial discretization $\Delta x$, this yields a restriction on the time step as $\Delta t \leq$ $\Delta x /|\alpha|$.

\section{Computational Experiments and Results}

In this section, we present three simulations to test robustness and accuracy of our model. We point out that the model parameters, especially the age dependent coefficient functions, 
require more careful research and hence, at this point, our current results are mainly useful in a qualitative sense. Nevertheless, even qualitative information on the dynamics of a disease as complicated as cholera can be helpful in gaining further insight. The following results consider a maximum age of $A=72$ years in the population and run for 24 weeks, which is a duration of approximately half a year. The code internally uses units of weeks for both age and time with an age resolution of 1 week and a time step of 1/50 weeks. Simulations in [1] report an earlier version of the studies for this model.

We specify all the model parameters when we discuss our simulations in the following subsections. However, we will point out an important model parameter here - the rate of waning of immunity. Since cholera vaccines require more frequent booster administration to children than adults, we model the rate of waning of immunity of humans at age $a$ by

$$
\omega(a)= \begin{cases}1 / 365, & a \leq 10 \text { years old } \\ 1 /(2 \cdot 365), & a>10 \text { years old }\end{cases}
$$

As we will see shortly, the rate of waning of immunity is important, because in particular, it influences our choice of initial conditions.

\subsection{The Reference Simulation with No Infected Population}

Suppose we have a pool of 10,000 humans distributed uniformly over the age range $0 \leq a \leq A$, none of whom are infected. Since there are no infected humans in this reference case, $I(a, 0)=0$ for all ages $a$ at $t=0$. Hence, all 10,000 humans are distributed to the susceptible and recovered categories. According to (5.1), it takes a year for a newborn baby to lose his or her immunity and become susceptible to cholera. Hence, we initialize everyone with age less than or equal to one year old in the removed category and everyone older than one year old in susceptible category. This translates to the initial conditions

$$
S(a, 0)= \begin{cases}0 & \text { if } 0 \leq a \leq 52 \text { weeks } \\ d & \text { if } a>52 \text { weeks }\end{cases}
$$

for the susceptible and

$$
R(a, 0)= \begin{cases}d & \text { if } 0 \leq a \leq 52 \text { weeks } \\ 0 & \text { if } a>52 \text { weeks }\end{cases}
$$

for the recovered populations, respectively. The numerical value of the age density $d$ in the initial conditions depends on the number of humans and the numerical resolution of the age variable: Numerically, using the age resolution in weeks, we will have a fixed density $d$ for each age $a$ for $0 \leq a \leq 52$, given by

$$
d=\frac{10,000(\text { humans })}{52(\text { week } / \text { year }) \times 72(\text { year })} \approx 2.67 \frac{\text { humans }}{\text { week }} .
$$

This gives the value of $d$ in the initial conditions for $S(a, 0)$ and $R(a, 0)$. With the age resolution of 1 week, thus at the initial time with constant density $d$, this gives $52 d \approx$ 


\begin{tabular}{|r|r|}
\hline Quantity & Value \\
\hline$\Lambda(a, t)$ & 0 \\
\hline$h(a, t)$ & 0.9 \\
\hline$u(a, t)$ & 0 \\
\hline$\beta_{L}$ & $\frac{1.5}{7}$ \\
\hline$\beta_{H}$ & $\frac{1.5}{7}$ \\
\hline$\kappa_{L}$ & $10^{6}$ \\
\hline$\kappa_{H}$ & $\frac{\kappa_{L}}{700}$ \\
\hline$\gamma_{1}$ & $\frac{1}{5}$ \\
\hline$\gamma_{2}$ & $\frac{1}{3}$ \\
\hline$\xi$ & $10^{9}$ \\
\hline$\eta$ & 0.1 \\
\hline$\chi$ & $120 /$ day \\
\hline$\delta_{L}$ & $\frac{1}{30}$ \\
\hline
\end{tabular}

Table 2: These are the values of the model parameters in all simulations except $\xi$ in Section 5.3. All units are the same as in Table 1.

139 humans of each age $a$. This is equivalent to saying that the 10,000 total humans are distributed to the ages 0 to 72 uniformly as 10,000/72 $\approx 139$ humans; these are distributed uniformly across the values in the age category with the finer numerical resolution of 1 week.

For the initial vibrio populations we make the following assumptions: $B_{L}(0)=0$, $B_{H}(0)=0$. These assumptions fix the choice of initial conditions in our system of differential equations.

Note that in our numerical results, we have a nonzero hydration strategy and have no antibiotic treatment strategy. Investigating both of these strategies as optimal controls will be considered in a future paper.

To model the disease related mortality rate, we use from Hartley et al. [22]

$$
\Delta(a)= \begin{cases}0.007, & a>10 \text { years old } \\ 0.032, & a \leq 10 \text { years old }\end{cases}
$$

Table 2 lists the values for the remaining model parameters.

In Figure 2, we depict the dynamics in the total population, susceptible population, infected population, and removed (recovered) population over time. As expected, with no infected people in the initial population, number of infected people remains zero at all times. Note that in this case, we see an increase in total population which is merely due to the positive balance in the rate at which babies are born versus natural death rate of people. We note here the decrease in the susceptible population, which is due to people who died of natural causes during the time-line of the simulation. Moreover, we see an increase in removed (recovered) population, which is due to the fact that newly born infants have immunity toward cholera, and it takes some time until they lose their immunity (which is governed by the rate of waning of immunity).

Even though there is no epidemic in this simulation, our model depicts more than just the population dynamics. The three-dimensional surface plots in Figure 3 show the advantages 
of our age-structured model even in this basic simulation. Each plot in Figure 3 shows the number of humans at an age $a$ in years at time $t$ in weeks; the color encodes the same information as the height of surface. The number of humans at a particular age is computed

by integrating each density, for instance, $S(a, t)$ from $a$ years to $a+1$ years by $\int_{a}^{a+1} S(\bar{a}, t) d \bar{a}$, representing the common understanding that humans from age $a$ to $a+1$ are considered $a$ years old. Notice that we use a resolution of 1 week in age, thus at the initial time with constant density $d$, this gives $52 d \approx 139$ humans of each age $a$. Comparing Figures 3 (c) and 3 (a) close to age 0 , we see how babies are born immune and then over time lose their immunity and become susceptible.

\subsection{Spread of an Epidemic with High Rate of Shedding of Cholera}

As before, we start with an initial population of 10,000 people. However, this time we will put one infected person of age 18 years in the initial population and use $\xi=10^{9}$. This is implemented in the code, with concentration units of humans per age by setting $I(a, 0)=0$ for $a<18$ and $a>19$ years and one uniform value $I(a, 0)>0$ for $18 \leq a \leq 19$ years such that

$$
\int_{18}^{19} I(a, 0) d a=1 .
$$

This initial condition on $I$ is interpreted as having one infected person of age 18 in the initial population. The numerical values for $I(a, 0)$ for $18 \leq a \leq 19$ years depend on the resolution of the age variable. For instance, with a numerical resolution of 1 week that we use, $I(a, 0)=1 / 52$ for $18 \leq a \leq 19$ years. The rest of the population of 9,999 humans is distributed in susceptible and removed categories similar to the previous simulation.

As before, the plots in Figure 4 depict over time, the total population, the susceptible population, the infected population, and the removed (recovered) population. We note the explosive nature of the spread of cholera in the plot of infected people where we see a high of about 5,000 infected people within the first couple of weeks of the epidemic. Qualitatively similar results were obtained in [22] using an ODE model. We also looked at the dynamics of $B_{H}$ and $B_{L}$ over time which are depicted in Figures 5 (a) and (b), respectively. They both show a peak within the first few days of the outbreak, but the less infectious vibrios have a greater accumulation in total area under the given curve. To take advantage of our PDE model, we can look at the model quantities in Figure 6 which show how the quantities change over time across different age groups. For example, in Figure 6, in the surface plot of the susceptible population, the height (the vertical coordinate) at point $(a, t)$ is the number of susceptible people of age $a$ at time $t$; the color encodes the same information as the height of surface. Since the susceptible and infected populations decrease, as expected, the recovered increase over time and age. Note due to infants, the recovered increase at a greater rate. This is assumed to occur due to a higher proportion of them having immunity from their mother's milk. Up to approximately age 72 , the susceptible population decreases exponentially. The infecteds have a peak at approximately 3 weeks. This coincides with the maximum height of the highly infectious and lesser infectious vibrios in Figure 5 (a) and (b). 


\subsection{Spread of an Epidemic with Low Rate of Shedding of Cholera}

In the previous simulation, we noted that our model reports a total of 5,000 infected people in less than two weeks; this points out the explosive nature of cholera [11]. One may simply ask, which aspect of the model captures this explosive nature of cholera. The answer to that question lies in the role played by hyperinfective vibrios $B_{H}(t),[22]$. Recall that in the system of differential equations describing our model, we had the ODE (2.5)

$$
\frac{d B_{H}}{d t}=\int_{0}^{A} \xi \eta I(a, t) d a-\chi B_{H}(t),
$$

where $\xi$ is the rate at which infected people shed hyperinfective vibrios into the aquatic environment. In the previous simulations, we had $\xi=10^{9}$. Making this number smaller implies that fewer hyperinfective vibrios are being released by people. To see what will happen in this case, we conducted our third simulation, where we let $\xi=10^{2}$. The remaining parameters are as listed in Table 2.

The results of this simulation reported in Figures 7, 8, and 9 show the dynamics of the epidemic in this case of lower rate of shedding of cholera with the inclusion of one infected of age 18. We see that when the hyperinfective vibrios play a less significant role, the result is a milder breakout of the disease. We see here that we get less than 1,500 infected people in over six weeks time, which is a much less severe case than that of our previous simulation.

With Figure 7, the dynamics in comparison to Figure 4 are different due to the decrease in $\xi$. We see an initial increase in the total population and a decrease between weeks 5 and 12 . The peak of the infected population occurs at roughly 5 weeks and decays to approximately zero by week 12. As expected, the susceptibles decrease in this interval. The removed population increases during this time. Yet, an interesting attribute is that the susceptible population increases slightly at the end of the time frame.

In Figure 8, we see that the peaks for the highly infectious vibrios shift by approximately 5 weeks with almost an $10^{7}$ decrease in its height. For the less infectious vibrios, the peak shifts in the same way. These graphs can help explain the shifts that occur in Figure 9 in relation to the higher shedding rate noted in Figure 6. In addition, the decrease in the vibrio volume directly effects the changes in the SIR curves due to the Michaelis-Menton terms involving $B_{H}$ and $B_{L}$.

In Figure 9, the shifts in the $B_{H}$ and $B_{L}$ peaks seen in Figure 8 make their effects known in the translations in the infected and susceptible peaks that occur later than in Figure 4. Another difference from Figure 4 is the increase in the susceptible population for a person under the age of 10 at the end of the time period. We can only speculate that this increase happens due to a longer time frame prior to the vibrio peak allowing more time for access to the disease, even though at lower levels of occurrence.

\section{Discussion}

An age-structured model can model the infection pathway of cholera more precisely, since the risk for contracting the disease depends on the age of a human. We see that introducing 
age as another independent variable entails solving a system of partial differential equations instead of simpler ordinary different equation systems; this introduces new challenges for the existence of a solution of the system and for the numerical method. We present our existence result for the PDE system using a fixed point argument. The reference simulation without any infected population reproduces the behavior of a population model. A simulation with a high rate of shedding of cholera produces results that show the same rapid speed of infection as traditional ODE models. To test the fidelity of the model and its implementation, a simulation with a low rate of shedding of cholera is contrasted, which has a more moderate spike of infections. This shows the central role played by the hyperinfective vibrios shed by infected and infectious people in the population, an important element pointed out in [22]. The results obtained by varying the rate of shedding motivate ideas on the different treatment options that may be possible with this age-structured model and its implementation. Combination treatments in an ordinary differential equation setting have been introduced and theoretically analyzed in [30]. The inclusion of treatment control strategies in an age-structured cholera model will be presented in future work.

\section{Acknowledgments}

This work was funded by the National Science Foundation DMS-0813563. Lenhart's support also included funding from the National Institute for Mathematical and Biological Synthesis NSF EF-0832858. We also thank David Hartley for useful discussions on this paper.

The co-authors Alexanderian and Gobbert started work on this problem with co-author Gaff as a consulting project through the Center for Interdisciplinary Research and Con-

sulting (www.umbc.edu/circ), while the first author was a graduate student in the Applied Mathematics program at UMBC. 

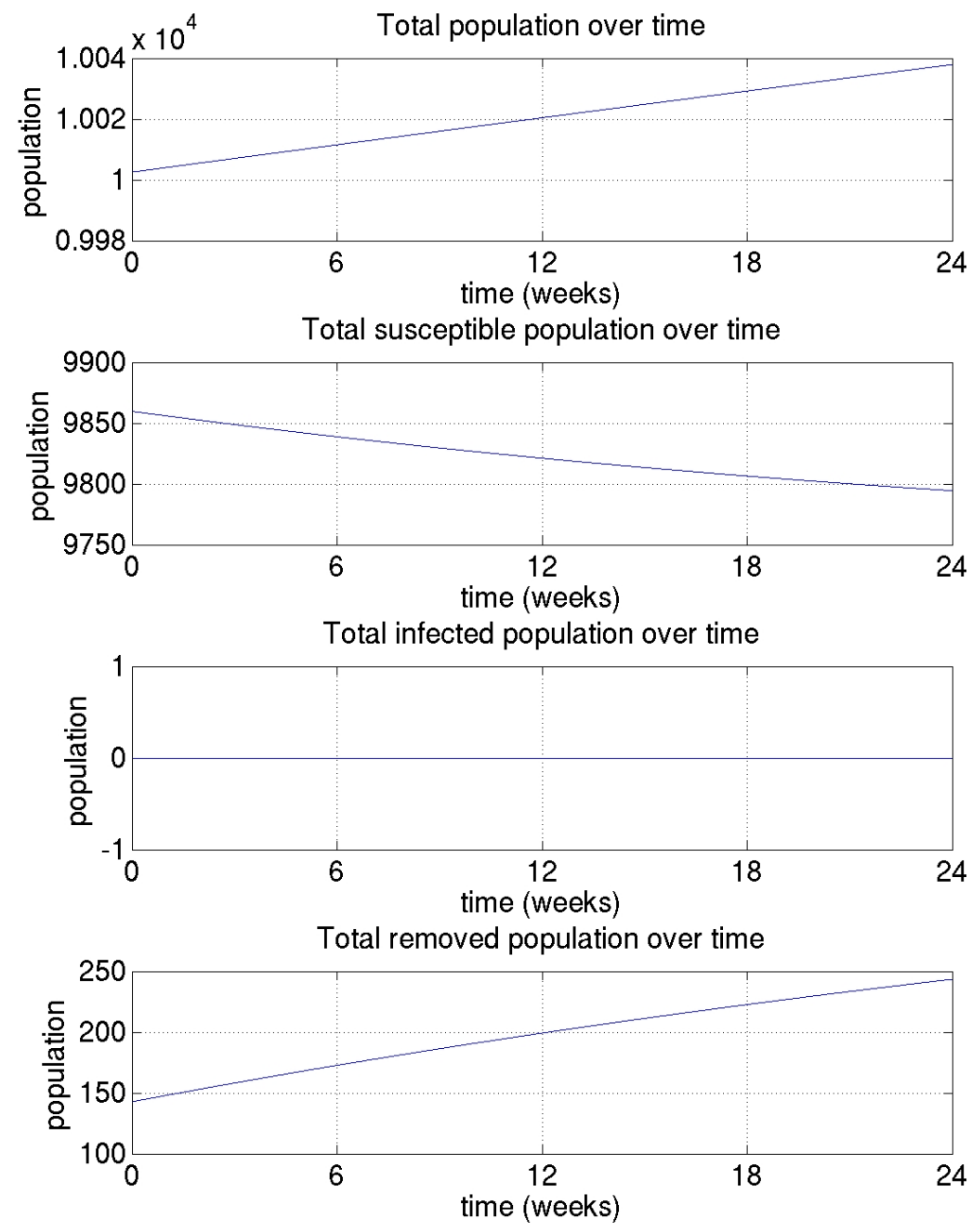

Figure 2: Reference simulation with no infected population: population dynamics over time. 

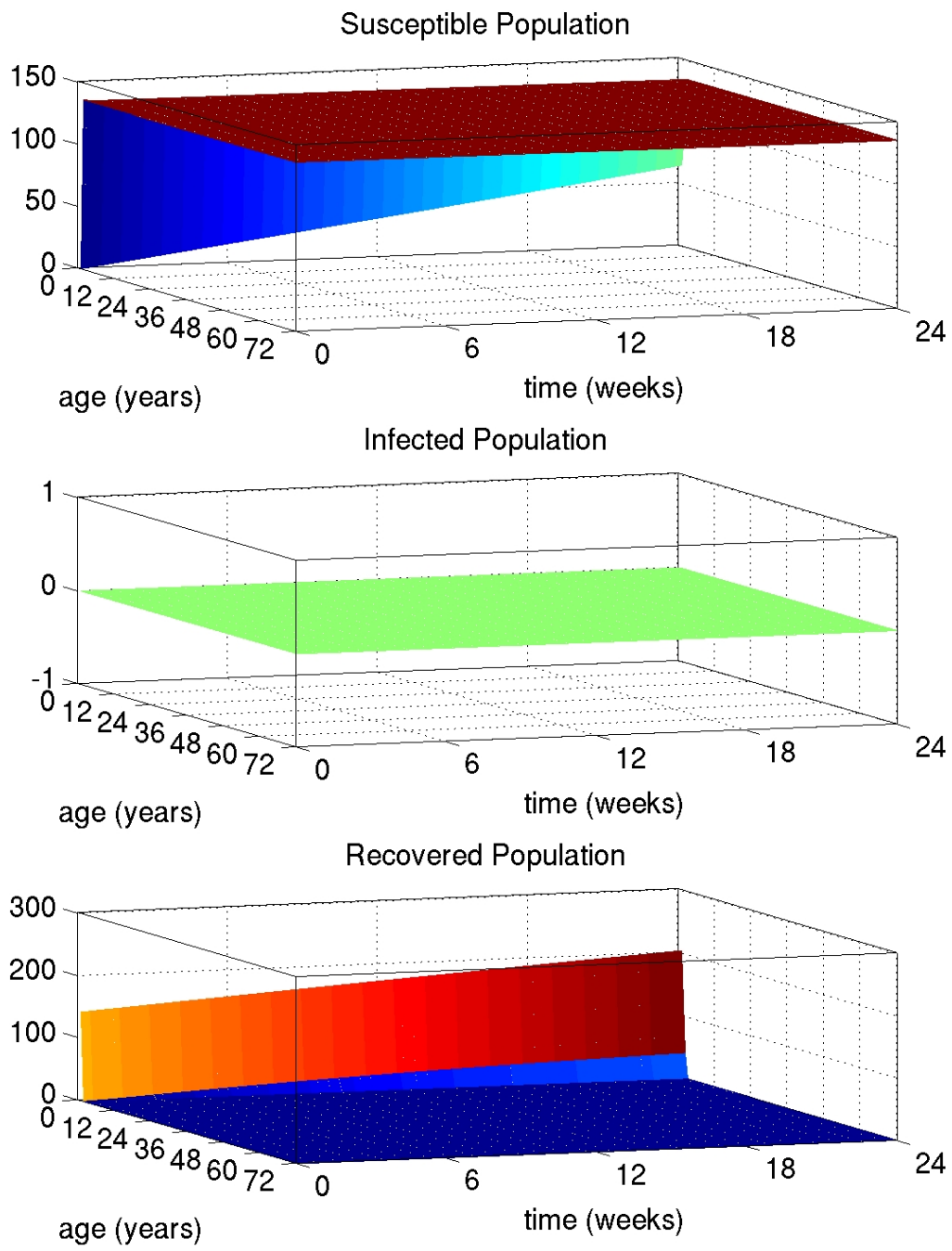

Figure 3: Reference simulation with no infected population: population dynamics as function of age and time. 


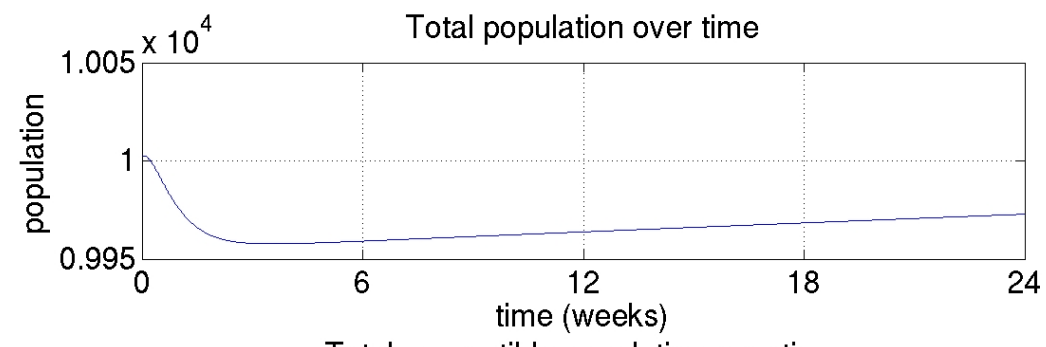

Total susceptible population over time

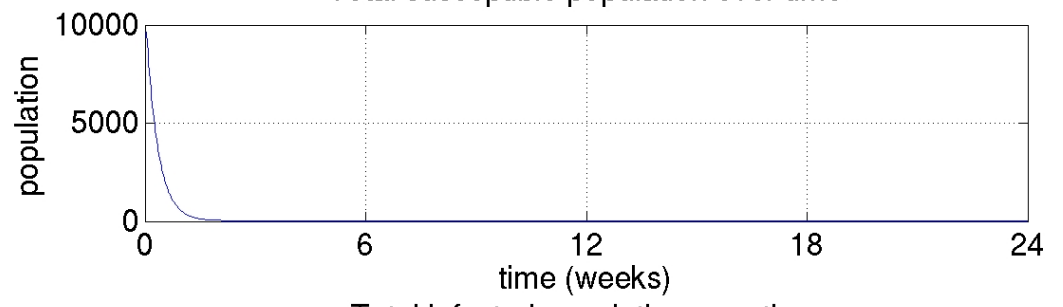

Total infected population over time

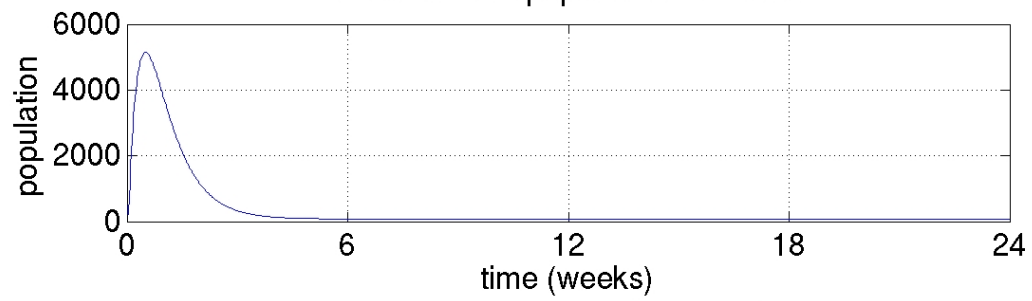

Total removed population over time

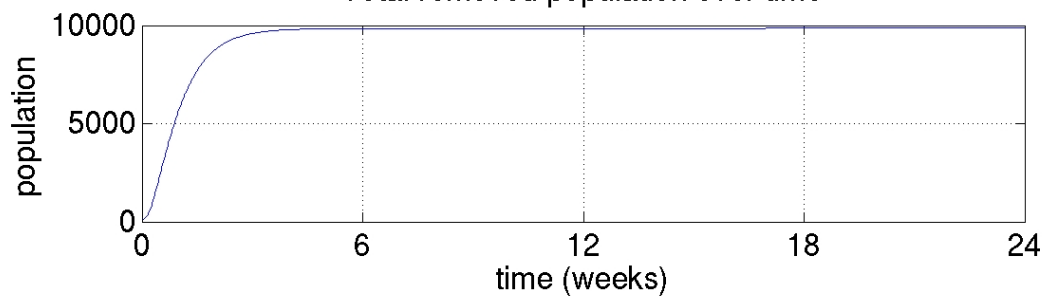

Figure 4: High rate of shedding of cholera: dynamics of the cholera epidemic over time. 


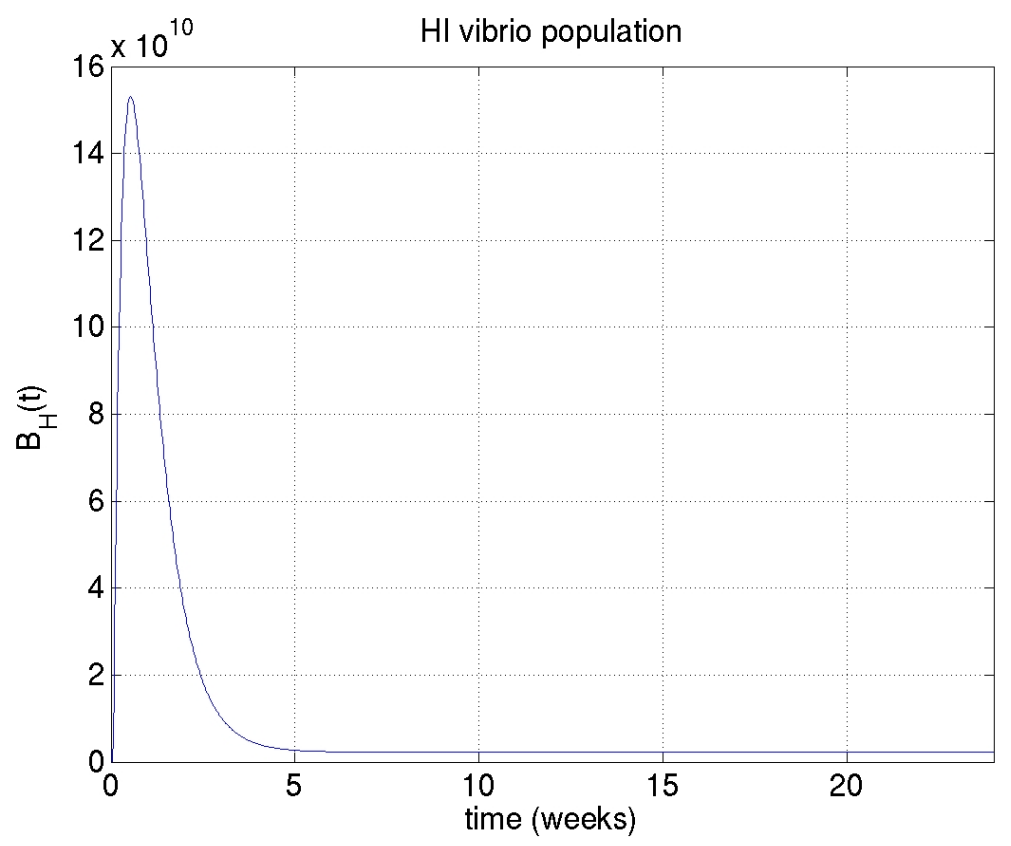

(a)

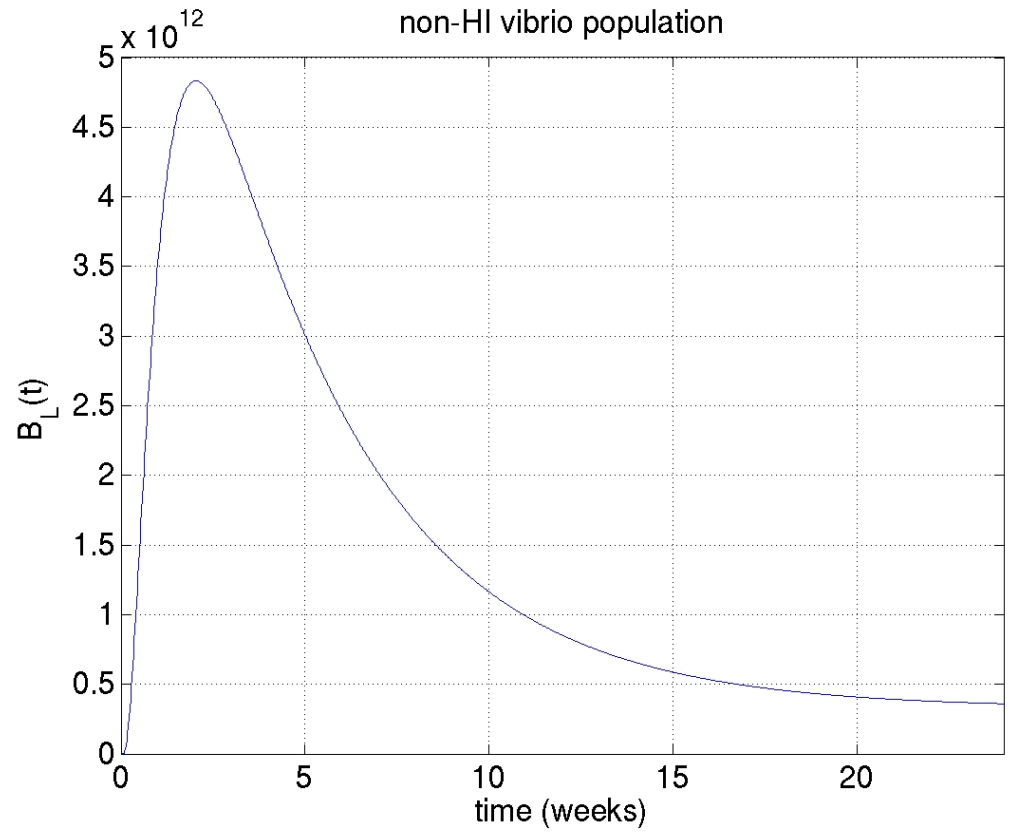

(b)

Figure 5: High rate of shedding of cholera: (a) $B_{H}$ over time, (b) $B_{L}$ over time. 

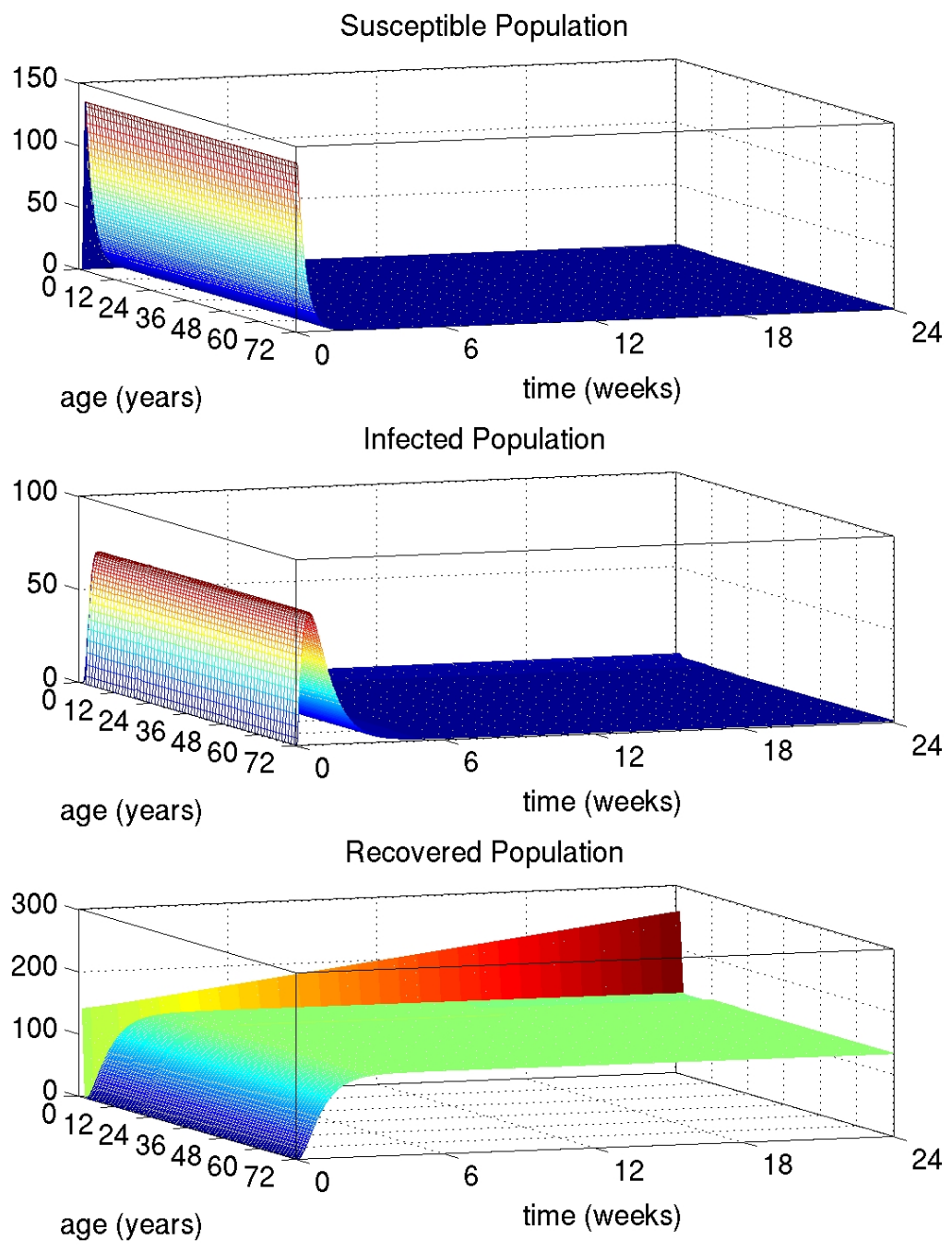

Figure 6: High rate of shedding of cholera: dynamics of the cholera epidemic as function of age and time. 

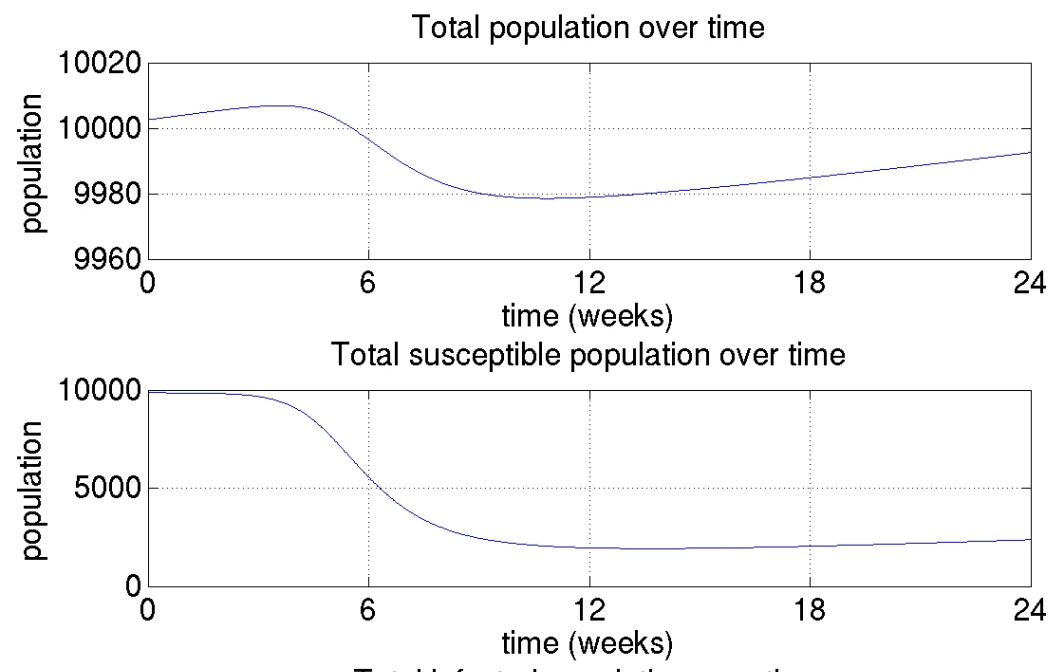

Total infected population over time

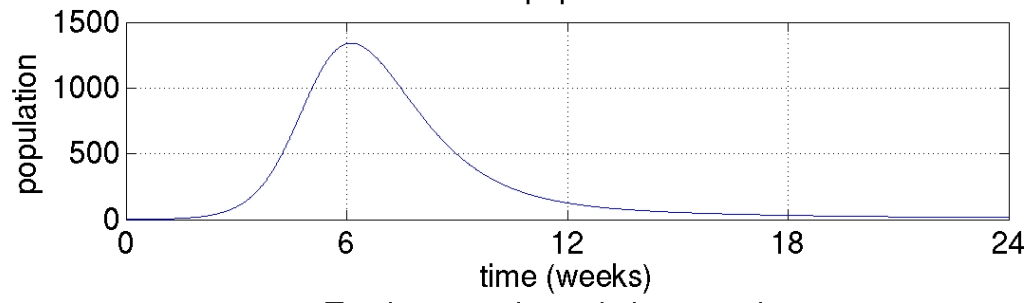

Total removed population over time

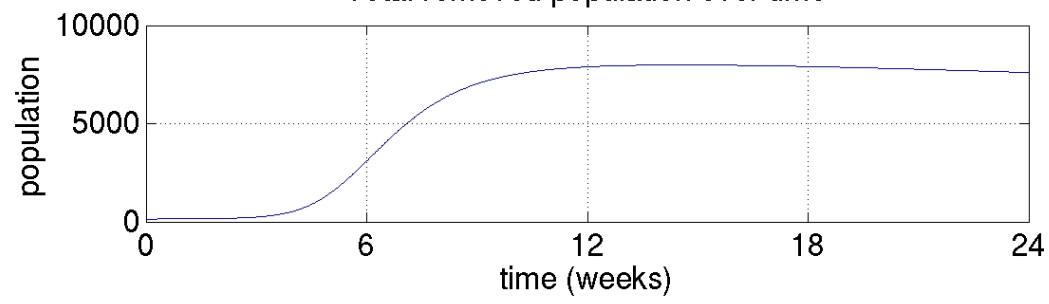

Figure 7: Low rate of shedding of cholera: dynamics of the cholera epidemic over time. 


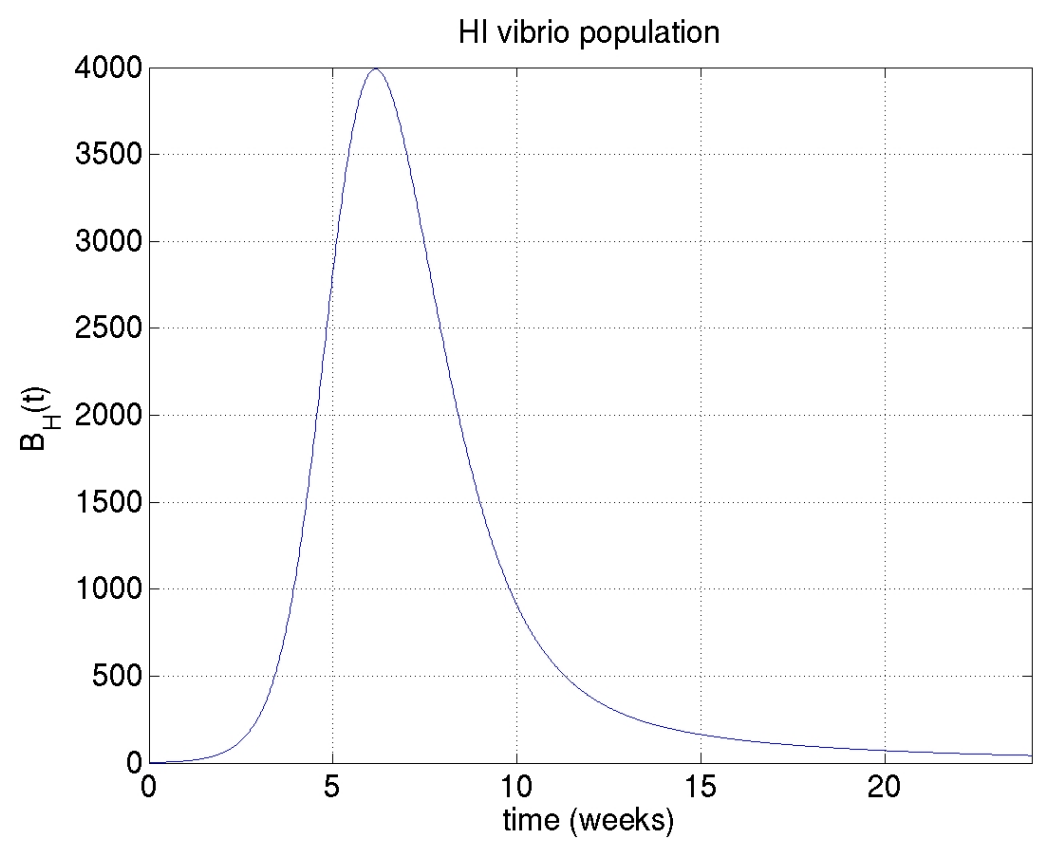

(a)

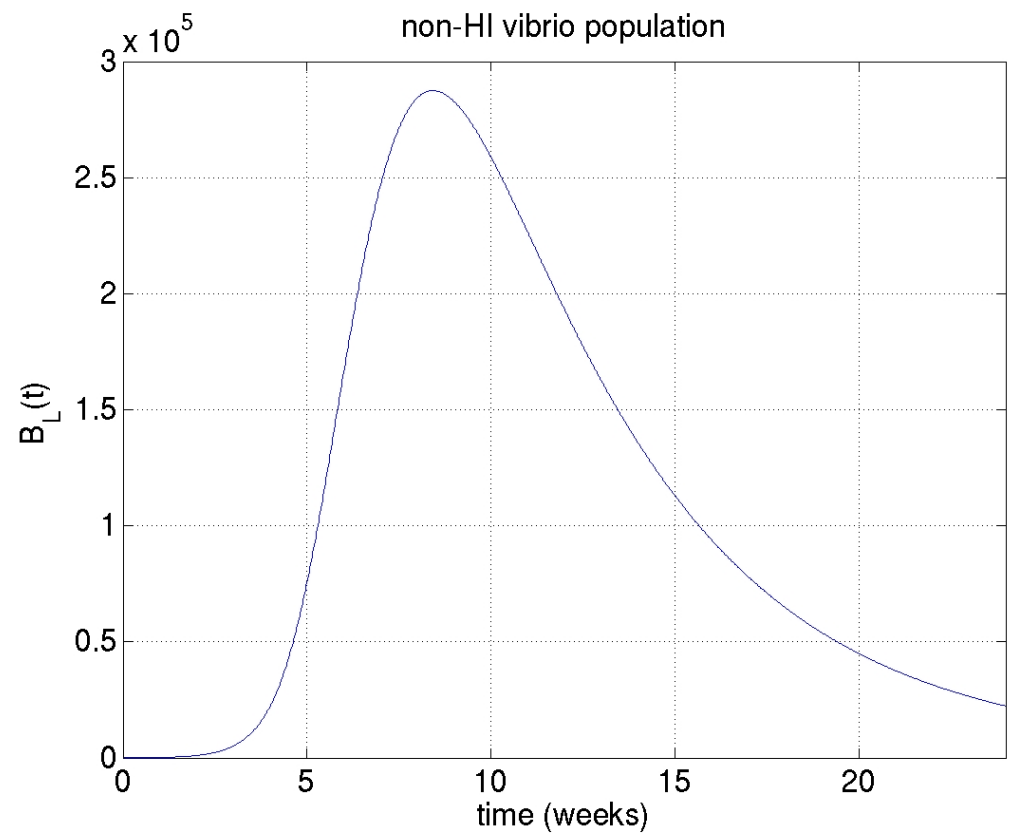

(b)

Figure 8: Low rate of shedding of cholera: (a) $B_{H}$ over time, (b) $B_{L}$ over time. 

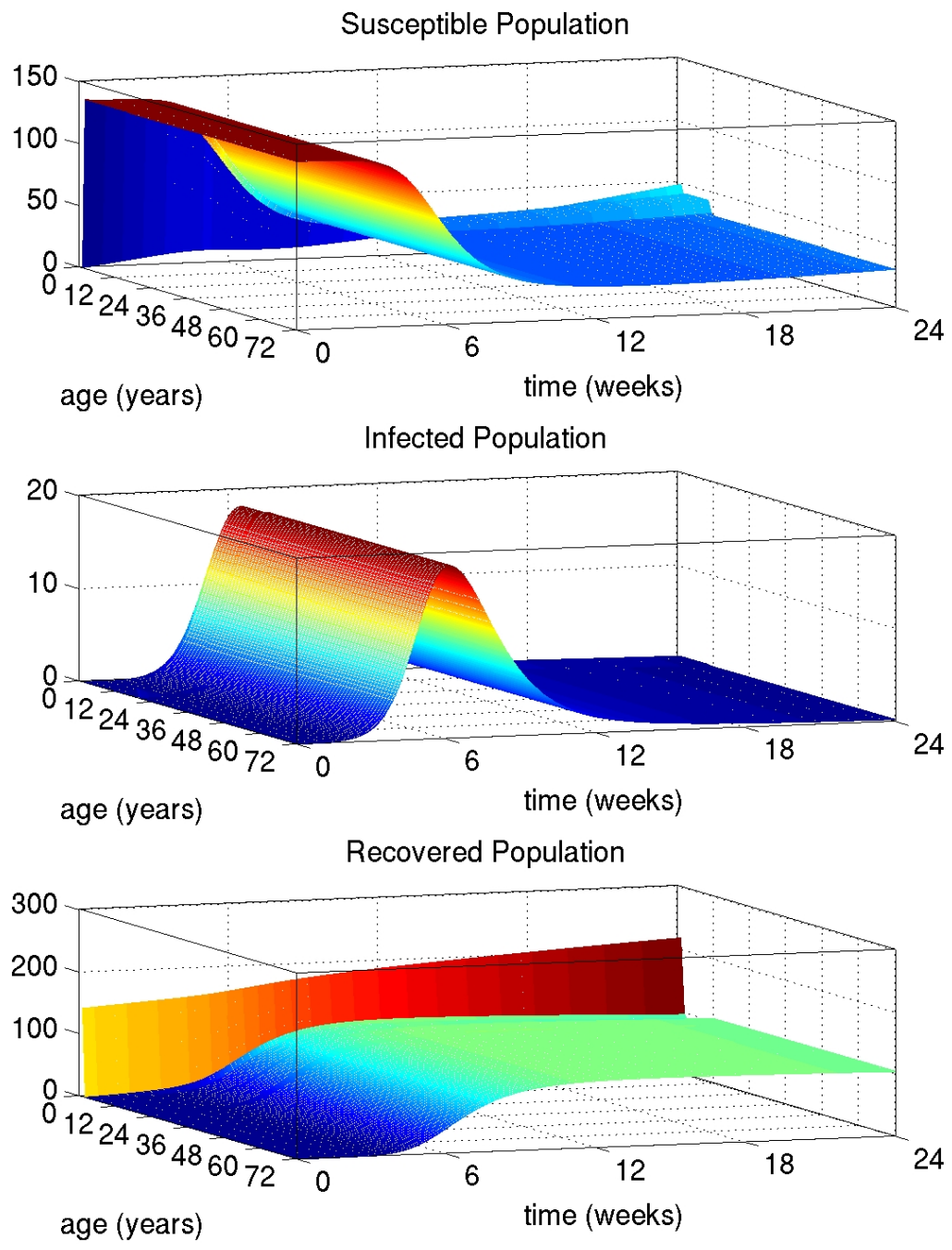

Figure 9: Low rate of shedding of cholera: dynamics of the cholera epidemic as function of age and time. 


\section{References}

[1] A. Agheksanterian, M.K. Gobbert, Modeling the spread of epidemic cholera: an agestructured model, Technical Report, Department of Mathematics and Statistics, University of Maryland, Baltimore County (2007), URL http://userpages.umbc.edu/ gobbert/papers/AlenGobbert2007Cholera.pdf.

[2] B. Ainseba, M. Langlais, On a population dynamics control problem with age dependence and spatial structure, J. Math. Anal. Appl. 248 (2) (2000), 455-474.

[3] S. Anita, Analysis and control of age-dependent population dynamics, Kluwer Academic Publishers, Boston, 2000.

[4] S. Anita, V. Capasso, A stabilization strategy for a reaction-diffusion system modelling a class of spatially structured epidemic systems (think globally, act locally), Nonlinear Analysis: Real World Applications 10 (2009), 2026-2035.

[5] V. Barbu, Mathematical methods in optimization of differential systems, Kluwer Academic Publishers, Dordrecht, 1994.

[6] V. Barbu and M. Iannelli, Optimal Control of Population Dynamics, J. Optim. Theory and Appl. 102 (1999), 1-14.

[7] A.L. Bishop, S. Schild, B. Patimalla, B. Klein, A. Camilli, Mucosal immunization with Vibrio cholerae outer membrane vesicles provides maternal protection mediated by antilipopolysaccharide antibodies that inhibit bacterial motility, Infect. Immun. 78 (10) (2010), 4402-4420.

[8] F. Brauer, Mathematical epidemiology is not an oxymoron, BMC Public Health 9 (Suppl. 1) (2009), S2.

[9] F. Brauer, Nonlinear age-dependent population growth under harvesting, Comput. Math. Appl. 9 (1983), 345-352.

[10] C.T. Codeço, Endemic and epidemic dynamics of cholera: the role of the aquatic reservoir, BMC Infect Dis. 1 (1) (2001).

[11] C.T. Codeço, F.C. Coelho, Trends in cholera epidemiology, PLoS Med. 3 (1):e42 (2006), $16-17$.

[12] J. Cushing, An Introduction to Structured Population Dynamics, SIAM, 1988.

[13] J. Cushing, M. Saleem, A competition model with age structure, Lecture Notes in Biomathematics, 44 (1982), 178-192.

[14] A. Ducrot, P. Magal, S. Ruan, Travelling wave solutions in multigroup age-structured epidemic models, Archive for Rational Mechanics and Analysis 195 (2009), 311-331. 
[15] L.K. Durham, I. Longini Jr., M.E. Halloran, J.D. Clemens, A. Hizam, and M. Rao, Estimation of Vaccine Efficacy in the Presence of Waning: Application to Cholera Vaccines. American Journal of Epidemiology 147 (10) (1998), 948-998.

[16] E.R. Eubanks, M.N. Guentzel, L.J. Berry, Evaluation of surface components of Vibrio cholerae as protective immunogens, Infect. Immun. 15 (2) (1977), 533-538.

[17] K.R. Fister, S. Lenhart, Optimal control of a competitive system with age-structure, J. Math. Anal. Appl. 291 (2004), 526-537.

[18] D.N. Georgiou, T.E. Karakasidis, J.J. Nieto, A. Torres, A study of entropy/clarity of genetic sequences using metric spaces and fuzzy sets, J. Theor. Biol. 267 (1) (2010), 95-105.

[19] R. Glass, A. Svennerholm, B. Stoll, M. Khan, K. M. Beleyet Hossain, M. Imdadui Hug, J. Holmgren, Protection against cholera in breast-fed children by antibodies in breast milk, N. Engl. J. Med. 308 (1983), 1389-1392.

[20] K. Gopalsamy, Age-specific coexistence in two-species competition, Math. Biosc. 61 (1982), 101-122.

[21] M.E. Gurtin, R.C. MacCamy, Nonlinear age-dependent population dynamics, Arch. Rational Mech. Anal. 54 (1974), 281-300.

[22] D.M. Hartley, J.G. Morris Jr., D.L. Smith, Hyperinfectivity: a critical element in the ability of v. cholerae to cause epidemics?, PLoS Med. 3 (1):e7 (2006), 63-69.

[23] H. Inaba, Mathematical analysis of an age-structured SIR epidemic model with vertical transmission, Discrete and Continuous Dynamical Systems Series B, 6 (1) (2006), 69-96.

[24] A. King, E.L. Ionides, M. Pascual, M. Bouma, Inapparent infections and cholera dynamics, Nature 454 (2008), 877-880.

[25] T. Kuniya, Global stability analysis with a discretization approach for an age-structured multigroup SIR epidemic model, Nonlinear Analysis: Real World Applications, In Press, doi:10.1016/j.nonrwa.2011.03.011.

[26] J. Lia, Dynamics of age-structured predator-prey population models, J. Math. Anal. Appl. 152 (1990), 399-415.

[27] D.S. Merrell, S.M. Butler, Host-induced epidemic spread of the cholera bacterium, Nature 417 (2002), 642-645.

[28] F.S. Mhalu, The problem of resistance to antimicrobial agents in the treatment and prevention of cholera. Proceedings of Nobel Conference 3 on acute enteric infections in children, New prospects for treatment and prevention. Holme, Holmgren, Merson, Molby (editors), 123, North Holland: Elsevier, 1981. 
[29] F.S. Mhalu, P.W. Mmari, J. Ijumba, Rapid Emergence of El Tor Vibrio Cholera Resistant to Antimicrobial Agents During First Six Months of Fourth Cholera Epidemic In Tanzania, The Lancet 313 (8112) (1979), 345-347.

[30] R. Miller Neilan, E. Schaefer, H. Gaff, K.R. Fister, S. Lenhart, Modeling optimal intervention strategies for cholera, Bulletin of Math. Biol. 1 (4) (2007), 379-393.

[31] W.H. Mosley, A.S. Benenson, R. Barui, A serological survey for cholera antibodies in rural east Pakistan. 2. A comparison of antibody titres in the innunized and control population of a cholera-vaccine field-trial area and the relation of antibody titre to cholera case rate, Bull. World Health Organ. 38 (3) (1968), 335-346.

[32] A. Naficy, M.R. Rao, C. Paquet, D. Antona, A. Sorkin, J.D. Clemens, Treatment and vaccination strategies to control cholera in sub-saharan refugee settings: a costeffectiveness analysis, JAMA 279 (7) (1998), 521-525.

[33] D. Pitkin, P. Actor, Immunity to Vibrio cholerae in the mouse I. Passive protection of newborn mice, Infect. Immun. 5 (4) (1972), 428-432.

[34] K. Qureshi, K. Molbak, A. Sandstrom, P. Kofoed, A. Rodrigues, F. Dias, P. Aaby, A. Svennerhom, Breast milk reduces the risk of illness in children of mothers with cholera: observations from an epidemic of cholera in Guinea-Bissau, The Pediatric Infectious Disease Journal 25 (12) (2006), 1163-1166.

[35] D. Saha, M.M. Karim, W.A. Khan, S. Ahmed, M.A. Salam, M.L. Bennish, Single-dose azithromycin for the treatment of cholera in adults, N. Engl. J. Med. 354 (23) (2006), $2452-2462$.

[36] M. Saleem, Predator-prey relationships: indiscriminate predation dynamics, J. Math. Biol. 21 (1984), 25-34.

[37] S. Schild, E.J. Nelson, A. Camilli, Immunization with Vibrio cholerae outer membrane vesicles induces protective immunity in mice, Infect. Immun. 76 (10) (2010), 4554-4563.

[38] J.B. Shukla, V. Singh, A.K. Misra, Modeling the spread of an infectious disease with bacteria and carriers in the environment, Nonlinear Analysis: Real World Applications, In Press, doi:10.1016/j.nonrwa.2011.03.003.

[39] J. Strikwerda, Finite difference schemes and partial differential equations, 2nd editon, SIAM, 2004.

[40] J.H. Tien, D.J.D. Earn, Multiple transmission parthways and disease dynamics in a waterborne pathgen, Bulletin of Math. Biol. 72 (6) (2010), 1506-1533.

[41] J.H. Tien, H.N. Poinar, D.S. Fisman, D.J.D. Earn, Herald waves of cholera in nineteenth century London, J. R. Soc. Interface 8 (58) (2011), 756-760. 
[42] A. Torres, J.J. Nieto, The fuzzy polynucleotide space: basic properties, Bioinformatics 19 (2003), 587-592.

[43] E. Venturino, Age-structured predator-prey models, Math. Modeling 5 (1984), 117-128.

[44] L. Wang, L. Chen, J.J. Nieto, The dynamics of an epidemic model for pest control with impulsive effect. Nonlinear Analysis: Real World Applications 11 (2010), 1374-1386.

[45] G. Webb, Theory of Nonlinear Age-Dependent Population Dynamics, Marcel Dekker, New York, 1985.

[46] World Health Organization, Cholera, Fact Sheet No. 107 (2008), URL http://www. who.int/mediacentre/factsheets/fs107/en/.

[47] H. Zhang, L. Chen, J.J. Nieto, A delayed epidemic model with stage-structure and pulses for pest management strategy. Nonlinear Analysis: Real World Applications 9 (2008), 1714-1726. 